\title{
Size-Controlled Synthesis and Optical Properties of Monodisperse Colloidal Magnesium Oxide Nanocrystals
}

\author{
Hoi Ri Moon, Jeffrey J. Urban, and Delia J. Milliron
}

Methods now exist to synthesize colloidal nanocrystals of a wide range of metals, chalcogenide and pnictide semiconductors, and some transition metal oxides. $^{[1]}$ The resulting products exhibit a spectrum of size-dependent properties and are solvent dispersible for processing and integration. For example, uniform polymer-nanocrystal composites can be prepared by casting from a co-solution of the two components to achieve composite materials with high refractive index, bright luminescence, or nonlinear optical properties. ${ }^{[2]}$ However, current solution-phase routes to alkaline earth metal oxide nanoparticles, such as $\mathrm{MgO}$ and $\mathrm{CaO}$, initially yield the hydroxide precursors $\left(\mathrm{Mg}(\mathrm{OH})_{2}\right.$ and $\left.\mathrm{Ca}(\mathrm{OH})_{2}\right)$ which must be calcined at high temperature to produce crystalline alkaline earth oxides. ${ }^{[3]}$ Unfortunately, this process results in uncontrolled grain growth and sintering of the nanoparticles into partially fused, random nanocrystalline networks. Efforts to break apart the networked powder and to disperse isolated nanocrystals using surfactants have resulted in aggregates $100 \mathrm{~nm}$ or larger with limited stability in dispersion. ${ }^{[4]}$ Alternatively, vapor phase methods such as chemical vapor deposition or combustion synthesis have been used to form $\mathrm{MgO}$ nanocrystals which are irrevocably substrate-bound. ${ }^{[5]}$ Here, we report the direct synthesis and optical characterization of colloidal magnesium oxide nanocrystals of narrow size distribution, tunable from $2-8 \mathrm{~nm}$ in diameter. The impact of bound surfactants on their optical properties is investigated through chemical exchange of their surface ligands. The development of solvent-processible, size-tunable alkaline earth oxide nanocrystals opens up new opportunities in rational catalysis, gas separations, and nano-optics.

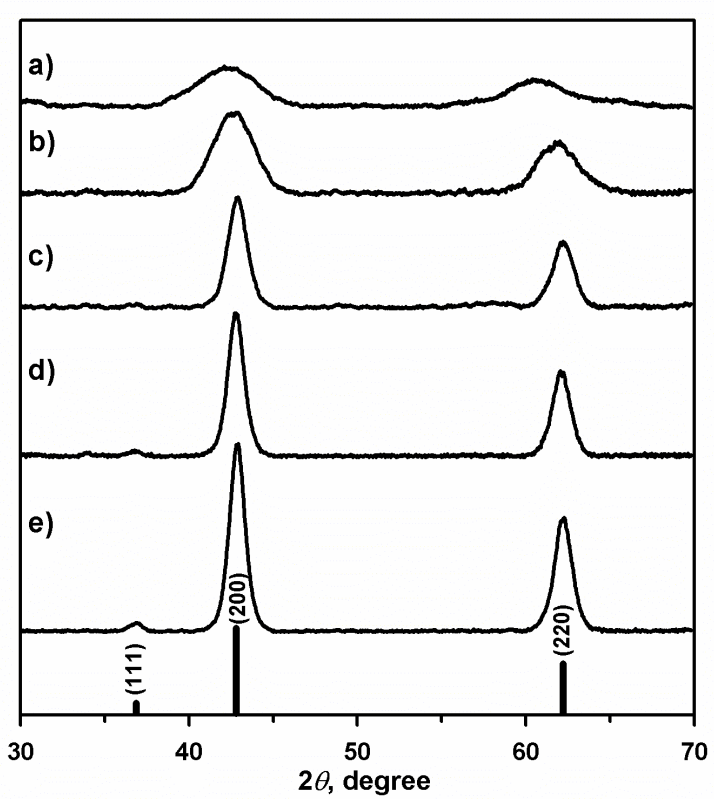

Figure 0 X-ray diffraction patterns of $\mathrm{MgO}$ nanocrystals synthesized with a) no added water, or b) $5 \mu \mathrm{L}$, c) $10 \mu \mathrm{L}$, d) $20 \mu \mathrm{L}$, and e) $40 \mu \mathrm{L}$ of added water to standard reaction conditions described in the text. Patterns are indexed according to cubic $\mathrm{MgO}$ (JCPDS 89-7746)

Solid films of alkaline earth oxides are commonly used in catalysis, either as a support for catalytically active metals such as Pt or Pd, or as an active catalytic surface. ${ }^{[3 a, 4,6]}$ Colloidal nanocrystals circumvent the inevitable reduction in surface area that occurs during calcination and introduce the possibility of carrying out catalysis in stable dispersions. ${ }^{[7]}$ Complementary to their established catalytic utility, alkaline earth oxide nanocrystals have potential optical applications. For example, $\mathrm{MgO}$ nanocrystals synthesized in the gas phase have been doped with calcium or transition metals to yield either broad blue emission or spectrally narrow photoluminescence, respectively. ${ }^{[5]}$ The bright blue emission we now report for colloidal $\mathrm{MgO}$ nanocrystals, not observed in bulk $\mathrm{MgO}$ crystals, offers an inexpensive, attractive alternative for optical applications.

$\mathrm{MgO}$ nanocrystals were synthesized from an organometallic precursor, bis(cyclopentadienyl)magnesium $\quad\left(\mathrm{Cp}_{2} \mathrm{Mg}\right), \quad$ and trioctylphosphine oxide (TOPO) in distilled benzyl ether. Briefly, a solution containing $308 \mathrm{mg} \mathrm{Cp} 2 \mathrm{Mg}(2 \mathrm{mmol})$ and $80 \mathrm{mg}$ TOPO $(0.2$ $\mathrm{mmol}$ ) in $4 \mathrm{~mL}$ benzyl ether was vigorously stirred and heated to $285^{\circ} \mathrm{C}$ for $2 \mathrm{~h}$ under $\mathrm{N}_{2}$ then immediately cooled to room temperature. Methanol was added to flocculate the nanocrystals, which were subsequently separated by centrifugation. The $\mathrm{MgO}$ nanocrystals could then be redispersed in a broad range of organic solvents, such as toluene and chloroform.

X-ray diffraction of the nanocrystals (Figure 1a) indicates that the product is single phase cubic $\mathrm{MgO}$ of the $\mathrm{Fm} \overline{3} \mathrm{~m}$ space group (JCPDS 89-7746). Remarkably, none of the common synthetic side products such as $\mathrm{Mg}(\mathrm{OH})_{2}$ or $\mathrm{MgCO}_{3}$ are observed. The positions of the (200) and (220) reflections are shifted to lower angle than bulk $\mathrm{MgO}$ and are noticeably broadened, clearly indicating the formation of $\mathrm{MgO}$

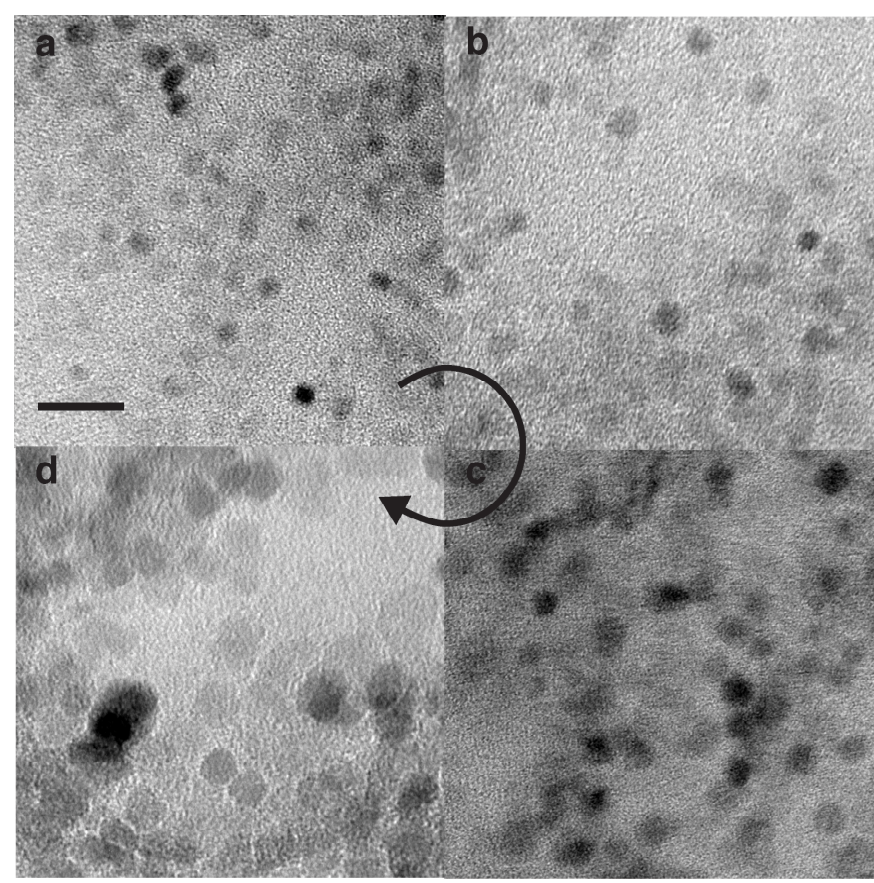

Figure 0 High resolution transmission electron microscopy (TEM) images of $\mathrm{MgO}$ nanocrystals oriented along the [100] (left) and (right) zone axes, and the corresponding Fourier transforms. The bars indicate $2 \mathrm{~nm}$ and, in the Fourier transforms, one spot of each symmetry related group is indexed according to the rock salt structure. nanocrystals of very small size and expanded lattice. The size of the 
nanocrystals is estimated by applying the Debye-Scherrer equation to the (200) reflection, correcting for instrumental broadening. The derived crystallite diameter is $2.1 \mathrm{~nm}$, somewhat smaller than that estimated by transmission electron microscopy (TEM), below.

The shape, size, and crystal structure of the MgO nanocrystals were confirmed by both low- and high-resolution TEM. We note that the low electron density of $\mathrm{MgO}$ (low atomic number) combines with the small size of these nanocrystals to severely limit the contrast in low-resolution imaging. Figure $2 \mathrm{a}$ shows a representative TEM image of the approximately spherical nanocrystals. Their diameter cannot unambiguously be quantitatively ascertained due to the low contrast, but is estimated to be $4.5+/-0.3 \mathrm{~nm}$ (a histogram of particle diameters is presented in Figure S1). The nanocrystals lie in random orientations on the carbon support film, resulting in particle-to-particle diffractive contrast variation in low-resolution TEM images and in the observation of different zone axes in high-resolution TEM images. Projections of the rock salt lattice along the [100] and [110] directions are clearly observed (Figure 3 ) and each particle is a single crystal, with no amorphous surface layer.

Size control of $\mathrm{MgO}$ nanocrystals was achieved by

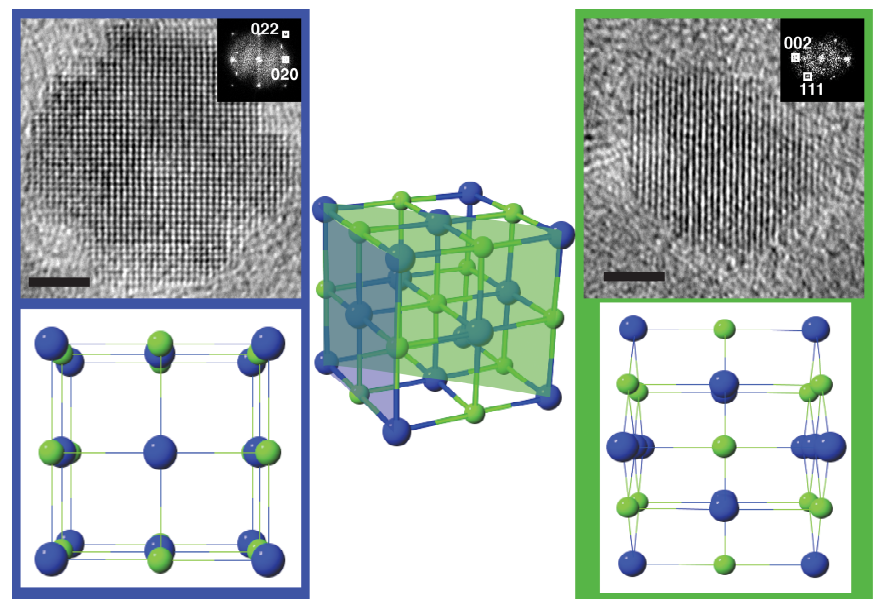

Figure 0 Low resolution transmission electron microscopy (TEM) images of $\mathrm{MgO}$ nanocrystals synthesized with a) no added water, or b) $5 \mu \mathrm{L}$, c) $10 \mu \mathrm{L}$, and d) $20 \mu \mathrm{L}$ of added water. The bar indicates $20 \mathrm{~nm}$. Nanocrystal diameter increases systematically with added water. systematically varying the reaction conditions. Addition of increasing, yet sub-stoichiometric, amounts of $\mathrm{H}_{2} \mathrm{O}$ to the reaction solution prior to heating yielded nanocrystals with increasingly larger diameters. The size derived from XRD peak broadening increased with the amount of $\mathrm{H}_{2} \mathrm{O}$ added: $2.1 \mathrm{~nm}(0 \mu \mathrm{L}), 3.0 \mathrm{~nm}(5 \mu \mathrm{L}), 6.4 \mathrm{~nm}(10 \mu \mathrm{L})$, and $7.6 \mathrm{~nm}(20 \mu \mathrm{L})$ (Figure 1a-d and Table S1). TEM results (Figures 2 and $\mathrm{S} 1$ ) are consistent with this trend and show that the spherical shape and narrow size distribution is maintained. The rounded shape of these nanocrystals differs from the highly faceted cubes formed by CVD. ${ }^{[5]}$ This difference, potentially significant for catalytic and optical properties, can be ascribed to the smaller size and organic ligand termination of the colloidal particles. For example, PbTe shares the rock salt crystal structure of $\mathrm{MgO}$ and colloidal $\mathrm{PbTe}$ is nominally spherical below $\sim 10 \mathrm{~nm}$ in diameter, forming faceted cubes at larger sizes. ${ }^{[8]}$ The size does not increase significantly further when more $\mathrm{H}_{2} \mathrm{O}(40 \mu \mathrm{L})$ is added (Figure 1e, deduced crystallite size $8.0 \mathrm{~nm}$ ), and the shape and size become less regular (Figure S2).

Although bulk, single crystalline $\mathrm{MgO}$ is a wide band gap insulator $\left(\mathrm{E}_{\mathrm{g}}\right.$ $>7 \mathrm{eV}$ ), optical transitions in the visible range have been observed in vapor phase synthesized $\mathrm{MgO}$ nanocrystals and assigned to surface states. ${ }^{[5]}$ These colloidal $\mathrm{MgO}$ nanocrystals exhibit a broad absorption band, which is centered around $325 \mathrm{~nm}$ and extends well into the visible range (Figure 4a). The absorption peak shifts slightly red at larger sizes, likely due to changing contributions from surface states associated with surface F-centers or with low coordinate oxygen anions, whose energies cover a broad sub-band gap range. ${ }^{[5,9]}$ The nanocrystals' photoluminescence (PL) is centered around $440 \mathrm{~nm}$, again shifting slightly with size (Figure 4b). Unlike CVD grown $\mathrm{MgO}$ nanocrystals, which required $\mathrm{Ca}^{2+}$ surface doping to luminesce brightly, these colloidal nanocrystals have a quantum yield of $15-19 \%$, falling off slightly at larger sizes (details reported in Table S2). Neither the absorption nor photoluminescence are consistent with any of reagents employed in the synthesis of the nanocrystals (Figure S3). We suggest that the large population of surface states on these small nanocrystals is responsible for the efficient blue emission, which is obvious to the naked

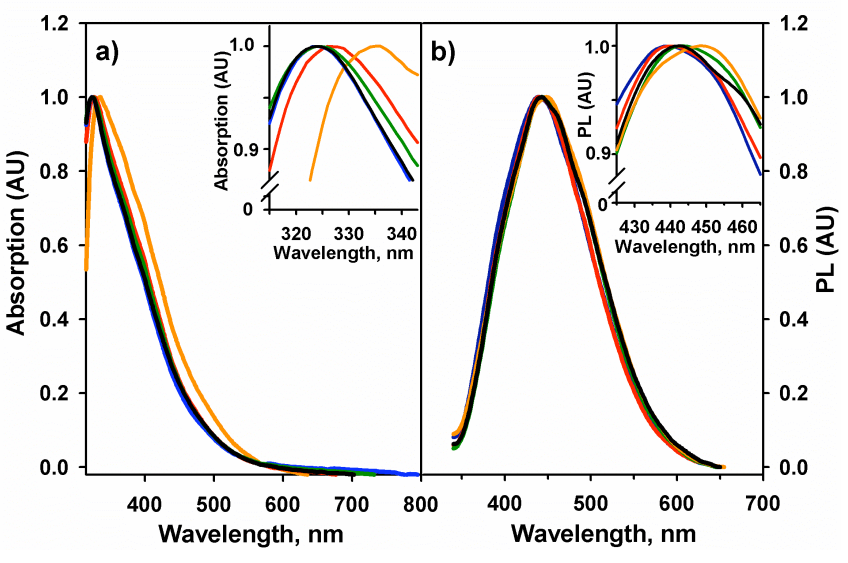

Figure 4 a) Optical absorption and b) photoluminescence (PL) spectra of $\mathrm{MgO}$ nanocrystals obtained by varying the amount of water in the reaction, in toluene dispersion.

Water amount: $0 \mu \mathrm{L}$ (black), $5 \mu \mathrm{L}$ (blue), $10 \mu \mathrm{L}$ (green), $20 \mu \mathrm{L}$ (red), and $40 \mu \mathrm{L}$ (orange). PL spectra were collected under excitation at $335 \mathrm{~nm}$. eye.

Because surface states are thought to dominate the optical properties, FTIR spectroscopy was used to discern the nature of the surfacecoordinating organic capping groups that facilitate dispersion of the nanocrystals in organic solvents. Based on the reagents included in the reaction solution, we consider three likely candidates: benzyl ether, TOPO, and tri( $n$-octyl)phosphine (TOP), produced as a side product. Benzyl ether is easily distinguished from TOPO or TOP by examining the $\mathrm{C}-\mathrm{H}$ stretch region of the FTIR spectra. The former is dominated by aromatic $\mathrm{C}-\mathrm{H}$ bonds at high stretching frequency, with a small contribution from methylene $\mathrm{C}-\mathrm{H}$ bonds at lower frequency (Figure $5 \mathrm{c}$ ). In contrast, TOP and TOPO are dominated by methylene $\mathrm{C}-\mathrm{H}$ stretching bands, with a smaller peaks arising from methyl C-H (Figure 5d) stretches. FTIR spectra for TOP and TOPO differ most notably in the strong $\mathrm{P}=\mathrm{O}$ stretching peak, appearing at $1144 \mathrm{~cm}^{-1}$ for neat TOPO (Figure $5 \mathrm{~d}$ ). This peak is, however, known to shift when TOPO binds to nanocrystal surfaces, ${ }^{[8]}$ and a similar peak might be anticipated for TOP bound to surface oxygen.

The as-synthesized $\mathrm{MgO}$ nanocrystals exhibit clear evidence of benzyl ether coordination in their FTIR spectrum. The C-H stretch region is dominated by aromatic bands, no $\mathrm{P}=\mathrm{O}$ stretching band is apparent, and the $\mathrm{C}=\mathrm{C}$ stretch at $1496 \mathrm{~cm}^{-1}$ is clearly visible (Figure $5 \mathrm{a}$ ). 
To confirm this assignment, ligand exchange was carried out to displace the as-synthesized surface ligands and to coat the surface specifically with either TOPO or benzyl ether. While the spectrum is effectively unchanged following ligand exchange with benzyl ether (Figure 5b), new peaks dominate the spectrum after TOPO exchange (Figure 5e). Methylene bands now dominate the $\mathrm{C}-\mathrm{H}$ stretch region and a peak at $1106 \mathrm{~cm}^{-1}$, assigned to surface coordinated $\mathrm{P}=\mathrm{O}$, ${ }^{[10]}$ appears. Weak aryl $\mathrm{C}-\mathrm{H}$ and $\mathrm{C}=\mathrm{C}$ stretching bands are evidence of residual benzyl ether. Thus, the as-synthesized $\mathrm{MgO}$ nanocrystals are coated in benzyl ether, and ligand exchange can be carried out to partially displace these ligands with TOPO. Furthermore, the PL quantum yield of the TOPO-exchanged nanocrystals was $50 \%$ reduced from the as-synthesized while benzyl ether exchange had a negligible effect on the PL. The efficient PL therefore depends not only on the population of surface states, but also on their interaction with the coordinated ligands. However, on the basis of these initial observations, we cannot discern the specific role that ligands play in the observed luminescence.

We have successfully synthesized monodisperse colloidal nanocrystals of the alkaline earth metal oxide $\mathrm{MgO}$, using added $\mathrm{H}_{2} \mathrm{O}$ to control the diameter in the $2-8 \mathrm{~nm}$ range. Very small $\mathrm{MgO}$ nanocrystals could be synthesized from the reaction of $\mathrm{Cp}_{2} \mathrm{Mg}$ with benzyl ether alone (Figure $\mathrm{S} 4$ ), but TOPO and $\mathrm{H}_{2} \mathrm{O}$ facilitate the production of nanocrystals with larger diameters. The resulting nanocrystals are single crystalline and exhibit size-dependent efficient blue luminescence. Their benzyl ether capping ligands facilitate dispersion in organic solvents and can be easily exchanged to tune surface chemistry and optical properties. These nanocrystals also

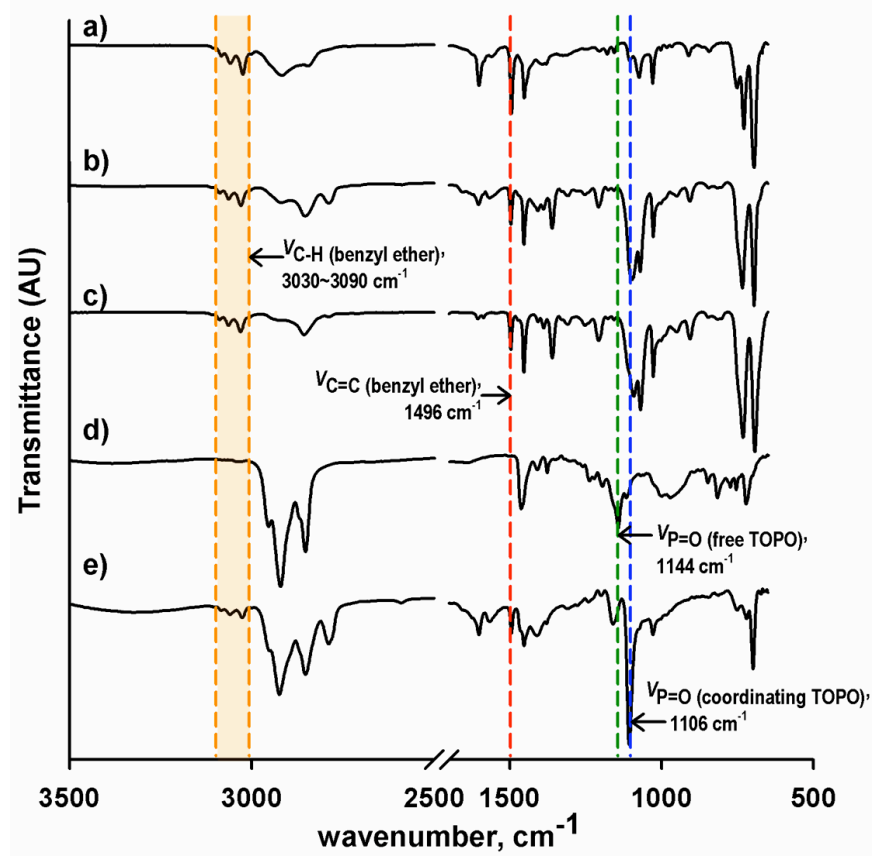

Figure 5 FTIR spectra of a) MgO nanocrystals assynthesized, and following ligand exchange with b) benzyl ether and with e) TOPO. For comparison, spectra of neat c) benzyl ether and d) TOPO are included. create new opportunities for rationally investigating and carrying out catalysis in colloidal dispersions.

\section{Experimental Section}

General Procedure for MgO Nanocrystal Preparation: Tri(n-octyl)phosphine oxide (TOPO) was dried at $130{ }^{\circ} \mathrm{C}$ for $3 \mathrm{~h}$ under vacuum, and benzyl ether was distilled over $\mathrm{CaH}_{2}$, then both were stored in a nitrogen glove box maintained at $<1 \mathrm{ppm} \mathrm{O}_{2}$ and $\mathrm{H}_{2} \mathrm{O}$. Bis(cyclopentadienyl)magnesium $\left(\mathrm{Cp}_{2} \mathrm{Mg}\right)(308 \mathrm{mg}, 1.99 \mathrm{mmol})$, TOPO $(80 \mathrm{mg}, 0.21 \mathrm{mmol})$, and benzyl ether $(4 \mathrm{~mL})$ were mixed and stirred magnetically at room temperature for $2 \mathrm{~h}$ under inert atmosphere. The reaction mixture was heated to $285^{\circ} \mathrm{C}$, and subsequently maintained at that temperature for $2 \mathrm{~h}$. The color of the solution changed from orange to dark brown. After cooling to room temperature, $\mathrm{methanol}(30 \mathrm{~mL})$ was added to the solution to yield a brown flocculate, which was separated from the solution by centrifugation (9000 rpm, $20 \mathrm{~min})$. The resultant brown product was redissolved in toluene $(4 \mathrm{~mL})$ and again flocculated with methanol $(20 \mathrm{~mL})$, and a powder comprised of MgO nanocrystals was finally obtained by centrifugation $(9000 \mathrm{rpm}, 20 \mathrm{~min})$. The recovered nanocrystals could be redispersed in toluene.

Effect of $\mathrm{H}_{2} \mathrm{O}$ on the formation of $\mathrm{MgO}$ nanocrystals. A mixture of $\mathrm{Cp}_{2} \mathrm{Mg}(308 \mathrm{mg}, 2.0 \mathrm{mmol})$, TOPO $(76 \mathrm{mg}, 0.20 \mathrm{mmol})$, and benzyl ether $(4 \mathrm{~mL})$, was stirred magnetically at room temperature for $2 \mathrm{~h}$ under inert atmosphere, as described above. To the solution were added 5 ( 0.28 $\mathrm{mmol}), 10(0.56 \mathrm{mmol}), 20(1.11 \mathrm{mmol})$, or $40 \mu \mathrm{L}(2.22 \mathrm{mmol})$ of $\mathrm{H}_{2} \mathrm{O}$, respectively, followed by heating to $285^{\circ} \mathrm{C}$. The isolation and recovery of nanocrystals proceeded as described above.

Ligand exchange with benzyl ether. As-prepared $\mathrm{MgO}$ nanocrystals dispersed in toluene $(1 \mathrm{~mL}, \sim 40 \mathrm{wt} \%)$ were added to benzyl ether (4 $\mathrm{mL}$ ), and the solution was stirred and heated at $80^{\circ} \mathrm{C}$ for $18 \mathrm{~h}$ under nitrogen. After cooling to room temperature, an excess of methanol (20 $\left.\mathrm{mL}\right)$ was added to induce flocculation of the nanocrystals, which were separated from the solution by centrifugation (9000 rpm, $30 \mathrm{~min})$. The resultant brown product could be redispersed in toluene.

Ligand exchange with TOPO. As-prepared MgO nanocrystals dispersed in toluene (1 mL, $40 \mathrm{wt} \%)$ were mixed with TOPO (140 mg, 0.35 mmol). After stirring for $18 \mathrm{~h}$ at $80^{\circ} \mathrm{C}$ under nitrogen, the solution was cooled to room temperature. The ligand-exchanged nanocrystals were flocculated by the addition of methanol $(10 \mathrm{~mL})$, and separated from the solution by centrifugation ( $9000 \mathrm{rpm}, 30 \mathrm{~min})$. The brown product could be redispersed in toluene.

Nanocrystal Characterization. The size and morphology of $\mathrm{MgO}$ nanocrystals were probed by high-resolution transmission electron microscopy (HRTEM) using a JEOL $2100 \mathrm{~F}$ operating at $200 \mathrm{kV}$. Samples for TEM analysis were prepared by drying a drop of toluene solution containing the nanocrystals on the surface of a carbon-coated copper grid. XRD patterns were obtained using a Bruker D8 Discover X-ray diffractometer with a general area detector diffraction system (GADDS) using Cu Ka radiation $(\lambda=0.154 \mathrm{~nm})$ from samples prepared by depositing precipitated powders on a quartz plate. An alumina plate (SRM 1976-National Institute of Standard Technology) powder standard pattern was obtained to determine the instrumental broadening, and peaks were fit to a Gaussian function. Infrared spectra were recorded on a PerkinEImer FTIR Spectrum One spectrophotometer. Absorption spectra were obtained with a Shimadzu UV-3600 UV-VIS-NIR Spectrophotometer. Photoluminescence spectra were recorded on a Jobin Yvon Horiba Fluorolog 3, and an integrating sphere employed to measure absolute quantum yields.

Keywords: Colloids $\cdot$ Synthesis Design $\cdot$ Sol-Gel Processes $\cdot$ Metal oxide $\cdot$ Nanocrystals $\cdot$ Blue Luminescence 
Acknowledgements: We thank Ms. S. Swanson and Dr. T. Topuria of the IBM Almaden Research Center for research contributions during the initial phases of this work. Work at the Molecular Foundry was supported by the Office of Science, Office of Basic Energy Sciences, of the U.S. Department of Energy under Contract No. DE-AC02-05CH11231. HRM is supported under B\&R code KC0202020, Hydrogen Storage program.

[1] a) J. Park, J. Joo, S. G. Kwon, Y. Jang, T. Hyeon, Angew. Chem. 2007, 119, 4714-4745; Angew. Chem. Int. Ed. 2007, 46, 4630-4660; b) S. G. Kwon, T. Hyeon, Acc. Chem. Res. 2008, 41, 1696-1709; c) Y. Yin, A. P. Alivisatos, Nature 2005, 437, 664-670; d) M. Niederberger, G. Garnweitner, Chem. Eur. J. 2006, 12, 7282-7302.

[2] a) L. Bakueva, S. Musikhin, M. A. Hines, T. -W. F. Chang, M. Tzolov, G. D. Scholes, E. H. Sargent, Appl. Phys. Lett. 2003, 82, 2895-2897; b) D. E. Fogg, L. H. Radzilowski, B. O. Dabbousi, R. R. Schrock, E. L. Thomas, M. G. Bawendi, Macromolecules 1997, 30, 8433-8439; c) P. P. Pompa, L. Matiradonna, A. Della Torre, L. Carbone, L. L. del Mercato, L. Manna, M. De Vittorio, F. Calabi, R. Cingolani, R. Rinaldi, Sens. Actuat. B-Chem. 2007, 126, 187-192; d) P. K. Sudeep, T. Emrick, Poly. Rev. 2007, 47, 155-163.

[3] a) K. Zhu, J. Hu, C. Kubel, R. Richards, Angew. Chem. 2006, 118, 7435-7439; Angew. Chem. Int. Ed. 2006, 45, 7277-7281; b) O. B. Koper, I. Lagadic, A. Volodin, K. J. Klabunde, Chem. Mater. 1997, 9, 2468-2480; c) P. P. Foderov, E. Tkachenko, S. V. Kuznetsov, V. V. Voronov, S. V. Lavrishchev, Inorg. Mater. 2007, 43, 502-504; d) W. Wang, X. Qiao, J. Chen, H. Li, Mater. Lett. 2007, 61, 3218-3220; e) C. Yan, D. Xue, J. Phys. Chem. B. 2005, $109,12358-12361$

[4] P. Jeevanandam, K. J. Klabunde, Langmuir 2003, 19, 5491-5495.

[5] a) S. Stankic, M. Sterrer, P. Hofmann, J. Bernardi, O. Diwald, E. Knozinger, Nano Lett. 2005, 5, 1889-1893; b) I. S. Altman, P. V. Pikhitsa, M. Choi, J. I. Jeong, H.-J. Song, I. E. Agranovski, T. E. Bostrom, Appl. Phys. Lett. 2003, 83, 3689-3691; c) I. S. Altman, P. V. Pikhitsa, M. Choi, H.-J. Song, A. G. Nasibulin, E. I. Kauppinen, Phys. Rev. B. 2003, 68, 125324.

[6] a) G. W. Wagner, P. W. Bartram, O. Koper, K. J. Klabunde, J. Phys. Chem. B. 1999, 103, 3225-3228; b) P. K. Stoimenov, V. Zaikovski, K. J. Klabunde, J. Am. Chem. Soc. 2003, 125, 12907-12913; c) J. Hu, K. Zhu, L. Chen, C. Kubel, R. Richards, J. Phys. Chem. C. 2007, 111, 12038-12044.

[7] R. Narayanan, M. A. El-Sayed, Nano Lett. 2004, 4, 1343-1348.

[8] J. E. Murphy, M. C. Beard, A. G. Norman, S. P. Ahrenkiel, J. C. Johnson, P. Yu, O. I. Micic, R. J. Ellingson, A. J. Nozik, J. Am. Chem. Soc. 2006, 128, $3241-3247$

[9] a) A. L. Shluger, P. V. Sushko, L. N. Kantorovich, Phys. Rev. B. 1999, 59, 2417-2430; b) V. I. Avdeev, G. M. Zhidomirov, J. Struct. Chem. 2003, 44, 918-926.

[10] a) J. E. B. Katari, V. L. Colvin, A. P. Alivisatos, J. Phys. Chem. 1994, 98, 4109-4117; b) A. G. Young, N. Al-Salim, D. P. Green, A. J. McQuillan, Langmuir 2008, 24, 3841-3849. 


\title{
Size-Controlled Synthesis and Optical Properties of
}

\section{Monodisperse Colloidal Magnesium Oxide Nanocrystals}

\author{
Hoi Ri Moon, Jeffrey J. Urban, and Delia J. Milliron*
}

The Molecular Foundry, Lawrence Berkeley National Laboratory, 1 Cyclotron Road, Berkeley, CA 94720 (USA)

*Email:dmilliron@lbl.gov
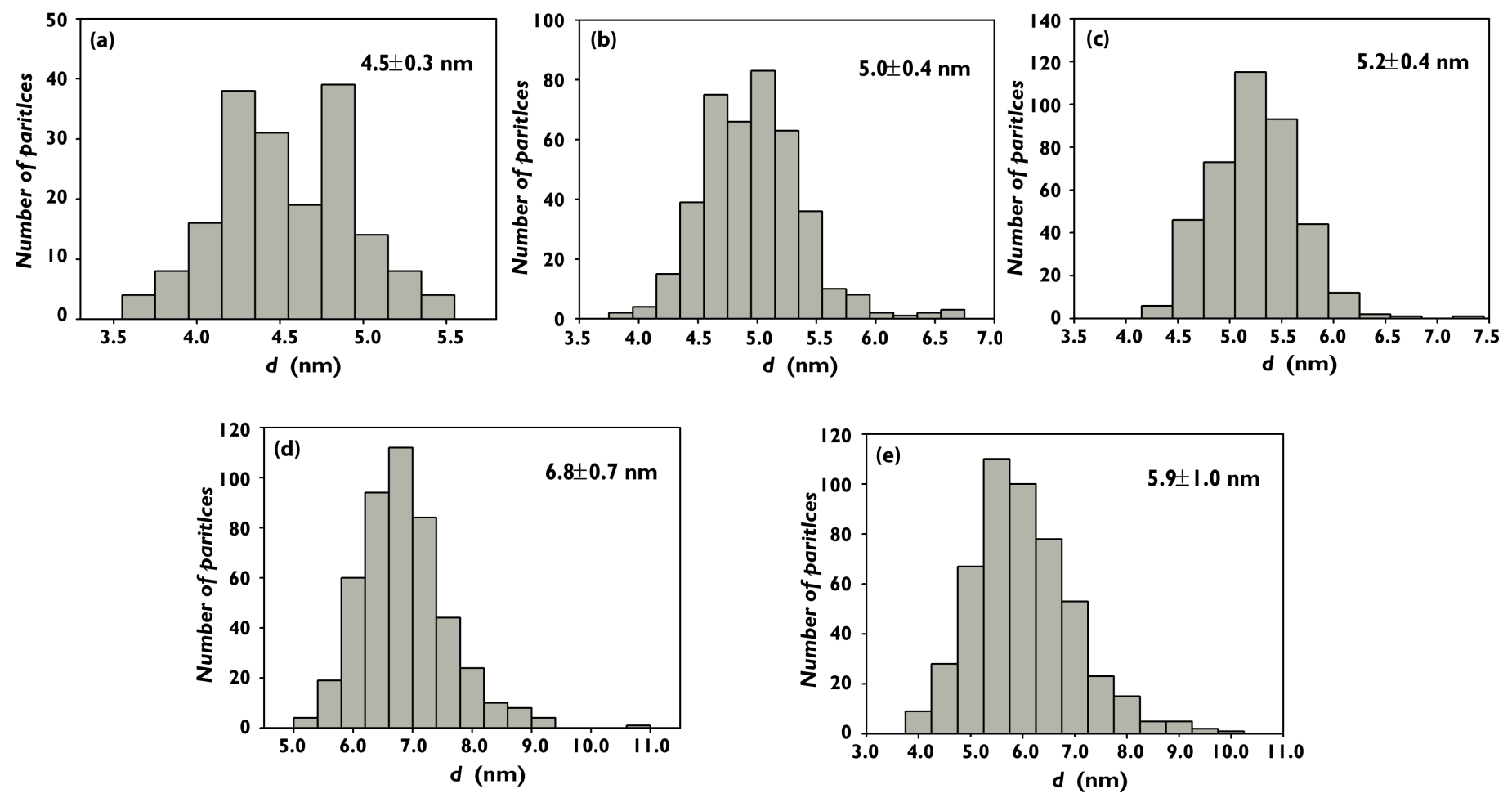

Figure S1. Particle size distribution histograms of $\mathrm{MgO}$ nanocrystals synthesized with a) no added water, or b) $5 \mu \mathrm{L}$, c) $10 \mu \mathrm{L}$, d) $20 \mu \mathrm{L}$, and e) $40 \mu \mathrm{L}$ of added water to standard reaction conditions described in the text. 


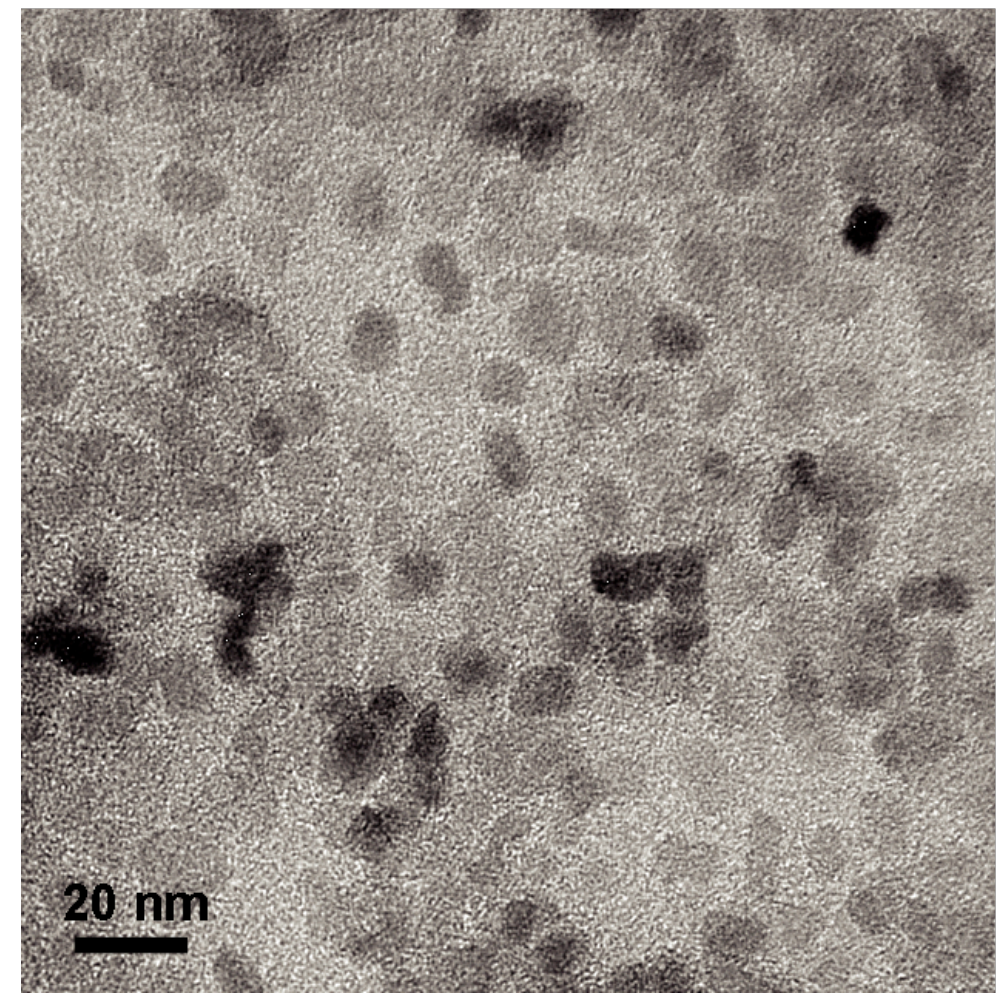

Figure S2. Low resolution transmission electron microscopy (TEM) image of $\mathrm{MgO}$ nanocrystals synthesized with $40 \mu \mathrm{L}$ of added water.

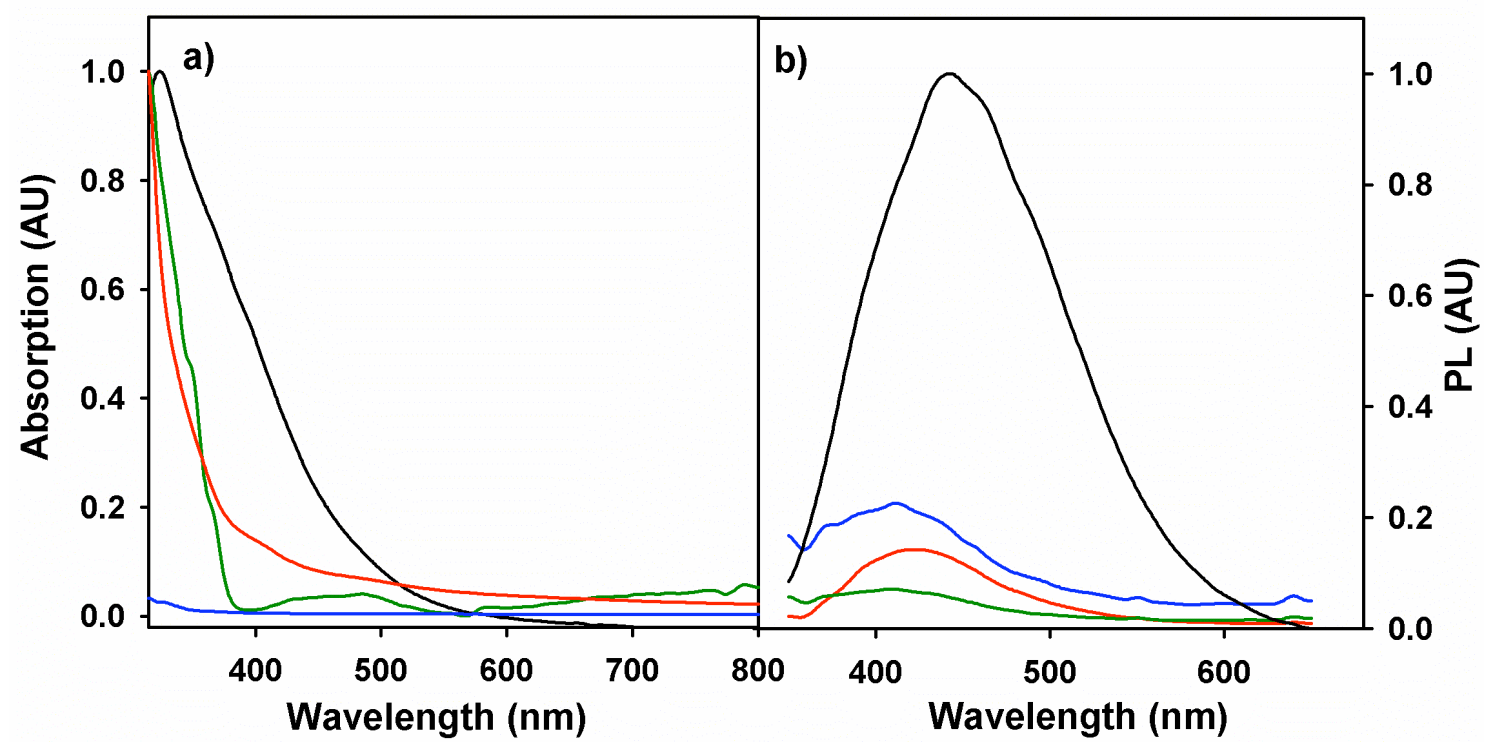

Figure S3. a) Optical absorption and b) photoluminescence (PL) spectra of the reactants used and $\mathrm{MgO}$ nanocrystals, in toluene. $\mathrm{MgO}$ nanocrystals (black), TOPO (blue), benzyl ether (green), and $\mathrm{Cp}_{2} \mathrm{Mg}$ (red). PL spectra were collected under excitation at $335 \mathrm{~nm}$, and were normalized to quantum yield. 


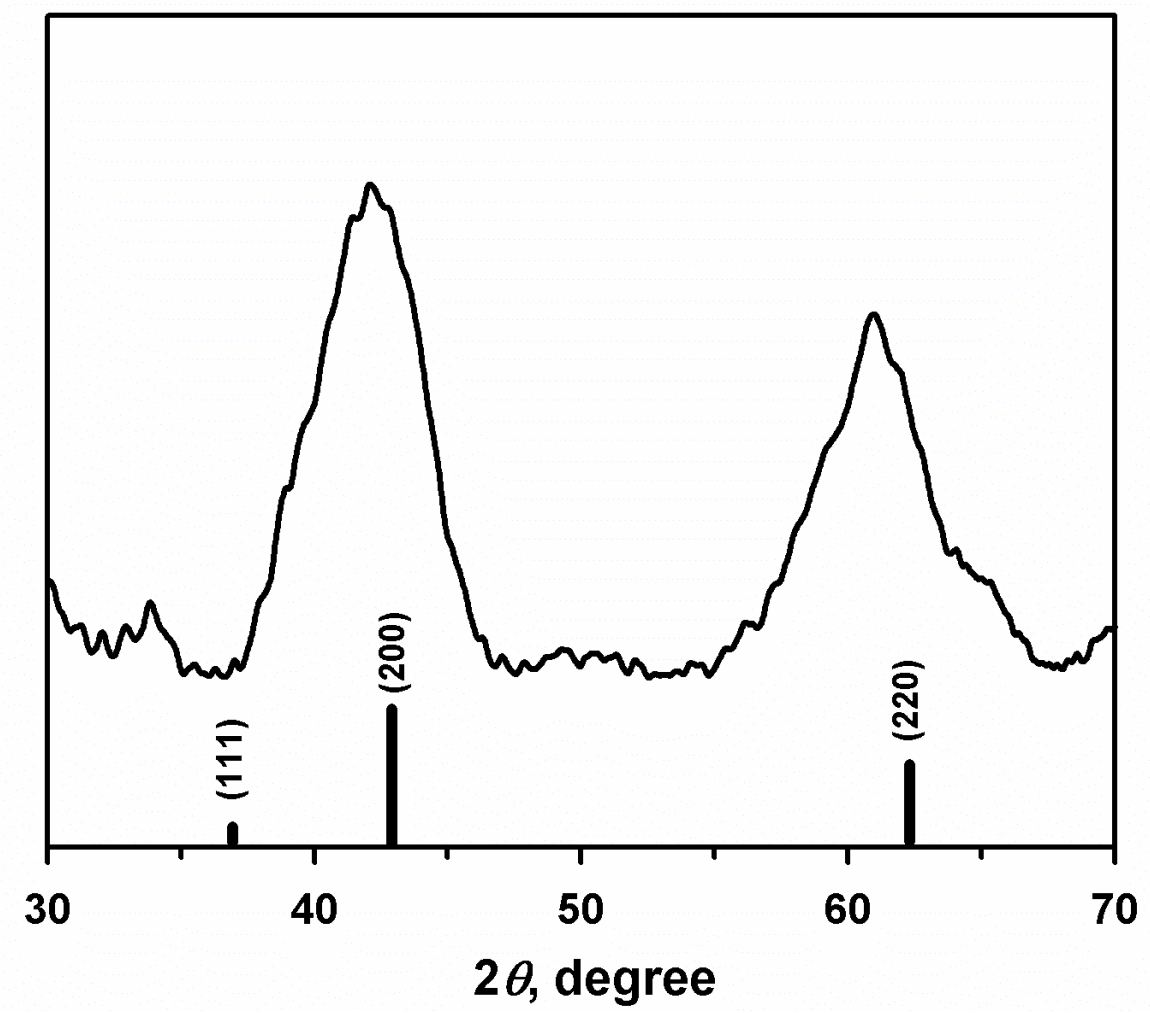

Figure S4. X-ray diffraction pattern of $\mathrm{MgO}$ nanocrystals synthesized from the reaction of $\mathrm{Cp}_{2} \mathrm{Mg}$ and benzyl ether without TOPO. 
Table S1. XRD results and the calculated particle sizes

\begin{tabular}{ccccc}
\hline $\begin{array}{c}\text { Molar ratio of } \\
{\left[\mathrm{Mg}^{2+}\right]:\left[\mathrm{H}_{2} \mathrm{O}\right]}\end{array}$ & $\begin{array}{c}\theta_{\max 1} \\
(200)\end{array}$ & $\begin{array}{c}\theta_{\max 2} \\
(220)\end{array}$ & $\begin{array}{c}\text { FWHM } \\
\text { Particle Size, }\end{array}$ & $\mathrm{nm}$ \\
\hline- & 42.23 & 60.56 & 4.16 & 2.1 \\
$8: 1$ & 42.63 & 61.94 & 2.84 & 3.0 \\
$4: 1$ & 42.88 & 62.22 & 1.40 & 6.4 \\
$2: 1$ & 42.79 & 62.04 & 1.18 & 7.6 \\
$1: 1$ & 42.86 & 62.28 & 1.14 & 8.0 \\
\hline
\end{tabular}

Table S2. UV-vis absorption and photoluminescence peak positions, ${ }^{[\mathrm{a}]}$ and quantum yields ${ }^{[\mathrm{b}]}$ on the basis of molar ratio of $\left[\mathrm{Mg}^{2+}\right]:\left[\mathrm{H}_{2} \mathrm{O}\right]$.

\begin{tabular}{cccccc}
\hline \multicolumn{2}{c}{ Compound } & & \multicolumn{2}{c}{$\lambda_{\max },[\mathrm{nm}]$} & \\
\cline { 1 - 2 } \cline { 5 - 6 } $\begin{array}{c}\text { Amount of } \\
\mathrm{H}_{2} \mathrm{O},[\mu \mathrm{L}]\end{array}$ & $\begin{array}{c}\text { Molar ratio of } \\
{\left[\mathrm{Mg}^{2+}\right]:\left[\mathrm{H}_{2} \mathrm{O}\right]}\end{array}$ & & Abs & PL & (\%) \\
\hline 0 & - & & & \\
\hline 5 & $8: 1$ & 324 & 442 & 15.5 \\
\hline 10 & $4: 1$ & 324 & 439 & 19.1 \\
\hline 20 & $2: 1$ & 326 & 440 & 15.7 \\
\hline 40 & $1: 1$ & 335 & 449 & 11.1 \\
\hline
\end{tabular}

[a] PL spectra were obtained under excitation at $335 \mathrm{~nm}$.

[b] Quantum yield was measured using an integrating sphere of 4 inch diameter as the sample compartment in the fluorometer. 